Original Article

\title{
Surgical Thrombectomy and Simultaneous Stenting for Deep Venous Thrombosis Caused by Iliac Vein Compression Syndrome (May-Thurner Syndrome)
}

\author{
Kimihiro Igari, MD, Toshifumi Kudo, MD, PhD, Takahiro Toyofuku, MD, PhD,
}

Masatoshi Jibiki, MD, PhD, and Yoshinori Inoue, $\mathrm{MD}, \mathrm{PhD}$

\begin{abstract}
Purpose: Iliac vein compression syndrome (May-Thurner syndrome) is characterized by left iliac vein obstruction secondary to compression by the right common iliac artery against the fifth-lumbar vertebra, which increases incidence of deep venous thrombosis (DVT). We treated the patients with DVT due to May-Thurner syndrome (MTS) by surgical thrombectomy and simultaneous stenting, and this study is to evaluate the outcomes of this procedure. Methods: From January 2009 to December 2011, a total of 8 patients underwent surgical thrombectomy with stenting. All patients were admitted for acute DVT involving the left iliofemoral segment, and diagnosed MTS. Patients were followed-up, and stent patency was assessed by means of duplex sonography.

Results: In all patients, the procedure was successful in achieving re-canalisation of the iliofemoral veins at the end of the operation. Perioperatively, there was no mortality and there was no case of clinically detected pulmonary embolism. Rethrombosis occurred within seven days of operation in 2 patients. During the follow-up period (mean; 16 months), 6 of 8 patients kept patent stents.

Conclusion: Venous thrombectomy with simultaneous stenting is a potent technique to treat acute iliofemoral DVT due to MTS. This technique can restore venous patency and provide relief of the acute symptoms.
\end{abstract}

Keywords: iliac vein compression syndrome, May-Thurner syndrome, deep venous thrombosis, surgical thrombectomy, stent placement

\section{Introduction}

Deep venous thrombosis (DVT) is a challenging situation. Its treatment should prevent thrombus extension,

Division of Vascular and Endovascular Surgery, Department of Surgery, Tokyo Medical and Dental University, Tokyo, Japan

Received: August 6, 2013; Accepted: October 8, 2013

Corresponding author: Kimihiro Igari, MD. Division of Vascular and Endovascular Surgery, Department of Surgery, Tokyo Medical and Dental University, 1-5-45 Yushima, Bunkyo-ku, Tokyo 113-8519, Japan

Email: igari.srg1@tmd.ac.jp

(C)2014 The Editorial Committee of Annals of Thoracic and Car-

diovascular Surgery. All rights reserved. pulmonary embolism, and long-term venous insufficiency, such as post-thrombotic syndrome (PTS). It is known to most clinicians that the standard recommendation for treatment of patients with acute DVT is antithrombotic therapy, which begins with heparin and is followed by oral anticoagulation with warfarin. However, antithrombotic therapy alone does not prevent long-term complications, mainly in cases of iliofemoral extension of the thrombus including iliofemoral obstruction cases. ${ }^{1-3)}$ Ziegler, et al. ${ }^{4)}$ showed that at a mean follow-up of 6 years after the onset of DVT, $82 \%$ of the patients presented with PTS.

May-Thurner syndrome (MTS) is a disease entity first described by May and Thurner in 1957 and is characterized 
Table 1 Indications and contraindications for vemous thrombectomy

\begin{tabular}{ll}
\hline Indications for thrombectomy & Contraindications for thrombectomy \\
\hline Iliofemoral DVT & Isolated DVT of infainguinal veins \\
Thrombus age $<14$ days & Thrombus age over 14 days and/or chronic deep venous insufficiency \\
Primary DVT & Recurrent DVT \\
\hline
\end{tabular}

DVT: deep venous thrombosis

by the occurrence of left common iliac vein (CIV) obstruction secondary to compression of the left common iliac vein by the right common iliac artery (CIA) against the fifth lumbar vertebra. ${ }^{5)}$ Classically, MTS was recognized as iliac vein compression syndrome. This anatomic variant can result in an increased incidence of DVT. Patients may go on to develop pulmonary emboli and symptoms of PTS, characterized by chronic edema, pain, hyperpigmentation, varicosities, and skin ulceration. Therefore, venous thrombectomy is a potential treatment option for DVT caused by MTS. ${ }^{2}$ To avoid re-thrombosis, venous outflow should be re-established ensuring that there is no residual stenosis in the left iliac veins. Even though the introduction of catheter-directed regional thrombolysis (CDT) has increased the rate of successful lysis; ${ }^{6)}$ however, the CDT results in a mean infusion time of 53 hours and major bleeding complications of $11 \%$ have been described. ${ }^{7)}$ Therefore, DVT due to MTS could be treated by surgical thrombectomy to avoid major bleeding complications and simultaneous stenting to restore the venous flow.

Herein, we present our experience with the simultaneous treatment for DVT due to MTS and evaluate our results of this treatment.

\section{Materials and Methods}

\section{Study population}

All protocols, surveys and consent forms were approved by the Institutional Review Board of Tokyo Medical and Dental University Hospital. Written informed consent was obtained from all subjects. Between January 2009 and December 2011, a total of 8 patients underwent transfemoral venous thrombectomy with simultaneous stenting. Indications as well as contraindications are summarized in Table 1. The study population comprised 8 patients (6 women), with a median age 75 years (range, 52-78 years).

\section{Preoperative evaluation}

All patients underwent screening for hypercoagulable disorders before treatment including screening for Protein $\mathrm{C}$, Protein S, antithrombin III deficiency, presence of lupus anticoagulant, and anticardiolipin antibody. Diagnosis of DVT was made by patient history, physical exam and duplex ultrasound. We used the color duplex ultrasound system (Xario ${ }^{\mathrm{TM}}$, Toshiba, Tokyo, Japan) with equipped with a linear array probe (frequency, 7.5 MHz) to investigate the lower limbs and a phased array probe (frequency, 3.5 MHz) to investigate the abdomen and pelvis. The initial diagnosis of DVT was made by duplex ultrasound. Subsequently, all patients underwent contrast enhanced computed tomography (CT) scans to assess the proximal extent of the thrombosis and emboli at the pulmonary arteries. The diagnose of MTS was made from the narrowing of the left CIV lumen due to the compression between the right CIA and the vertebra by CT scanning (Fig. 1A). The proximal and distal extent of the thrombus in the pelvic region as well as the other patient characteristics are summarized in Table 2. The table shows that a DVT involving all venous segments from the pelvis to the calf was present in 50\% cases. The mean time interval between the onset of symptoms and treatment by venous thrombectomy was 7 days (range, 4 to 14 days). In all cases, CT showed the compression of the left CIV against the spine and pelvic brim by the right CIA.

\section{Hybrid procedure}

All procedures were performed in the operating room. We performed these procedures under general $(n=4)$ or local $(n=4)$ anesthesia, with the assistance of a cellsaver auto-transfusion device (SORIN XTRA ${ }^{\circledR}$, SORIN GROUP, Milan, Italy). Preoperatively, temporary inferior vena caval filter (Neuhaus Protect ${ }^{\mathrm{TM}}$, Toray Medical, Tokyo, Japan) was placed to protect against embolization during intervention. ${ }^{8)}$ Filters were deployed in standard fashion from the contralateral groin at the level of L2/L3 under fluoroscopic guidance. After that, the common femoral vein (CFV) was approached through an oblique incision in the groin. After adequate dissection of the vessel, $5000 \mathrm{IU}$ of heparin was given intravenously and the CFV was clamped distally. A transverse venotomy was placed on the anterior surface of the CFV and the iliac veins were then cleared of thrombus with the help 


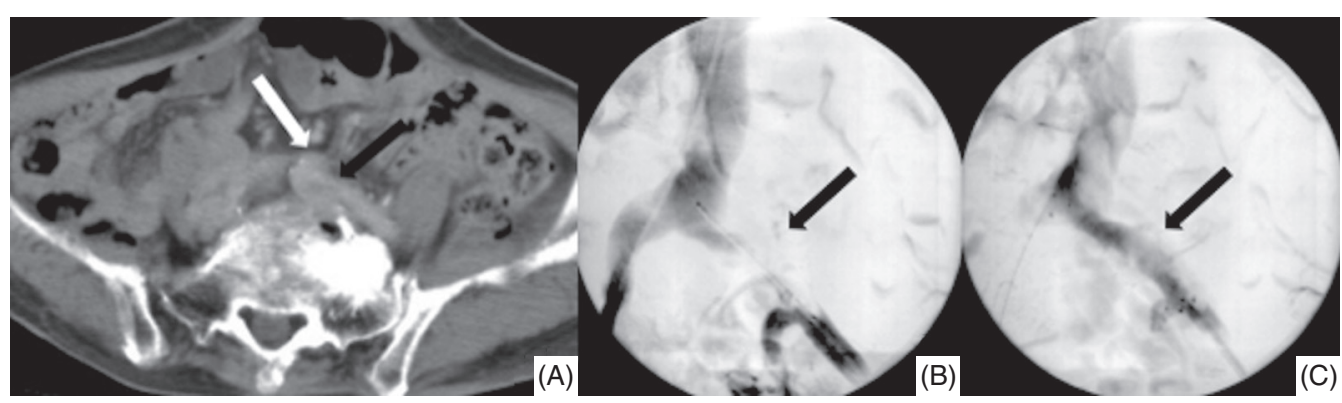

Fig. 1 (A) The diagnosis of May-Thurner syndrome by computed tomography. The left common iliac vein (black arrow) was compressed between the right common iliac artery (white arrow) and the vertebra. (B) Intraoperative angiography showed the occluded left common iliac vein (black arrow) was catheterized with a 0.035 -inch guidewire through the obstructive lesion to the inferior vena cava. (C) After the stent deployment (black arrow), the left common iliac venous flow was restored.

Table 2 Patients background

\begin{tabular}{cccclc}
\hline Patient No. & Gender & Age & Comorbidity & Location of thrombus & Duration from onset (days) \\
\hline 1 & F & 78 & Malignancy Protein S deficiency & Iliac - femoral & 6 \\
2 & M & 71 & - & Iliac - femoral & 4 \\
3 & M & 66 & - & Iliac - calf & 11 \\
4 & F & 65 & Malignancy & Iliac - calf & 6 \\
5 & F & 74 & Malignancy & Iliac & 5 \\
6 & F & 52 & - & Iliac - femoral & 4 \\
7 & F & 75 & - & Iliac - calf & 7 \\
8 & F & 78 & - & Iliac - calf & \\
\hline
\end{tabular}

F: female; M: male

of a Fogarty catheter. A 6 or 7 Fr sheath was then introduced through the transverse venotomy, and venography by using contrast medium injection was performed to visualize the obstructive lesion of left iliac vein. The vein was catheterized with a 0.035 -inch guidewire through the obstructive lesion to the inferior vena cava (IVC), and angioplasty was performed with 4-7 mm angioplasty balloon. After that, $12 \mathrm{~mm}$ self-expanding stents (E-LUMINEXX ${ }^{\circledR}$, BARD, Murray Hill, New Jersey, USA) was placed and deployed over the stenotic segment, followed by post dilatation with $8-10 \mathrm{~mm}$ angioplasty balloon (Fig. 1B and 1C). Thrombotic material below the inguinal ligament was evacuated by manual compression. Fogarty catheter was not applied distally from the femoral vein to avoid the destroying venous valve function. We have also used isolated intra-operative thrombolysis of the distal venous segments. This technique included the intravenous injection of $480000 \mathrm{IU}$ urokinase and $5000 \mathrm{IU}$ heparin diluted in $500 \mathrm{ml}$ Ringer solution via a foot vein. During the infusion, the CFV remained clamped to prevent anticoagulant being mixed into the systemic circulation, in addition, a tourniquet was placed on the thigh as high as possible and inflated to $300 \mathrm{mmHg}$ to force the anticoagulant solutions into the deep veins of lower extremity. The isolated intra-operative thrombolytic solution was left to act for 30 minutes, and during this time the thrombectomy of the iliac veins was carried out. The clamp on the CFV was moved proximally to the venotomy and the clots from the distal venous segments were flushed out through the opening in the CFV. This was aided by massaging the thigh and calf. When the venous blood was retrieved with cell-saver, we retransfused after processing. ${ }^{9)}$ At the end of this procedure, temporary IVC filter was removed if there was no capture of large thrombi detected by the venography. When a large thrombus was detected in the filter, we performed postoperative systemic thrombolytic therapy with another temporary IVC filter placement via a jugular vein for a week, after that, we removed it.

\section{Postoperative treatment}

Postoperatively, all patients received full anticoagulation, first with heparin and then with warfarin for at least 6 months.

\section{Follow-up}

Follow-up was scheduled at 1, 3, 6, and 12 months and then biannually. It included clinical examination and 
Table 3 Operating procedure

\begin{tabular}{|c|c|c|c|c|c|}
\hline Patient No. & Anesthesia & $\begin{array}{c}\text { Stent (diameter } \times \\
\text { length }[\mathrm{mm}], \text { location })\end{array}$ & $\begin{array}{l}\text { Agent of } \\
\text { thrombolysis }\end{array}$ & $\begin{array}{l}\text { Intraoperative } \\
\text { complication }\end{array}$ & Additional procedure \\
\hline 1 & $\mathrm{~L}$ & $12 \times 80, \mathrm{CIV}$ & - & - & - \\
\hline 2 & $\mathrm{~L}$ & $12 \times 60, \mathrm{CIV}$ & - & - & - \\
\hline 3 & $\mathrm{G}$ & $12 \times 40, \mathrm{CIV}$ & + & - & - \\
\hline 4 & G & $12 \times 60$ & + & $\begin{array}{l}\text { Branch of EIV } \\
\text { ruptured }\end{array}$ & $\begin{array}{l}\text { Coil embolization to the } \\
\text { ruptured branch of EIV }\end{array}$ \\
\hline 5 & $\mathrm{~L}$ & $\begin{array}{c}12 \times 80,12 \times 60, \\
12 \times 60, \text { CIV }- \text { EIV }\end{array}$ & - & - & - \\
\hline 6 & $\mathrm{~L}$ & $12 \times 60, \mathrm{CIV}$ & - & - & - \\
\hline 7 & G & $12 \times 40, \mathrm{CIV}$ & + & - & - \\
\hline 8 & G & $12 \times 40, \mathrm{CIV}$ & + & - & - \\
\hline
\end{tabular}

L: local; G: general; CIV: common iliac vein; EIV: external iliac vein

duplex scanning. Swelling was assessed by measuring the circumference of both legs. Skin changes such as reticular or varicose veins, trophic skin changes and ulceration were documented. Duplex scanning was performed with a special focus on patency of stents, patency and competence of deep and superficial veins, and signs of PTS.

\section{Results}

\section{Patients characteristics (Table 2)}

All patients presented with unilateral left leg swelling, pain and iliofemoral DVT. Thrombus involvement was isolated to the iliofemoral venous segments in 4 patients and extended throughout the iliofemoropopliteal venous segments in the remaining 4 patients. In addition to anatomical obstruction, one patient had Protein S deficiency, and 3 patients had malignant disease ( 2 patients with colorectal carcinoma and one patient with breast carcinoma). The remaining 4 patients had no clotting abnormalities, and no personal or large familial history of DVT was found in these patients.

\section{Operating procedure (Table 3)}

In all patients, the procedure was successful at restoring patency of the pelvic veins at the end of the operation. No perioperative death or pulmonary embolism occurred. All 8 patients were treated with self expanding nitinol stents after thrombectomy and angioplasty of the iliac vein thrombus and stenosis. Ten stents $(12 \mathrm{~mm}$ in diameter and $40-80 \mathrm{~mm}$ long) were used. One patient were treated with three stents due to the residual stenosis of the left common to external iliac vein with thrombus. The mean number of stents for treating the lesions per patient was 1.2. All stents were placed above the inguinal ligament. Isolated intra-operative thrombolysis was performed in
4 cases (50\%). Even though the blood transfusion was needed intraoperatvely in 3 patients (37\%), we had no further blood transfusion in these three cases postoperatively.

\section{Early complications and concomitant procedures (Tables 3 and 4)}

Three patients (37\%) had intraoperative or early postoperative complications after operation. One patient had perforation of the small branch of the external iliac vein intraoperatively and treated by coil embolization. One patient with history of previous left femoral DVT had rethrombosis at day 5 , but refused a second operation. One patient had rethrombosis at day 7, and rethrombectomy was performed with good result. All patients were discharged with warfarin therapy and elastic stockings. Seven of 8 patients $(87 \%)$ were relieved from their symptoms dramatically at the discharge.

\section{Follow-up (Table 4)}

Mean follow-up time is 16 months (range, $1-42$ months). One patient died from carcinomatosis one month after her discharge with a patent stent, and no had pulmonary embolism during the entire follow-up. During the follow-up period, one patient experienced asymptomatic rethrombosis of the previously reopened venous segment, but further operation was not performed. Duplex ultrasonography revealed that 6 of 8 patients had no abnormal findings in any part of the venous system. Two stents permanently occluded. We could find no evidence of progressive stenosis in the patent stents during the follow-up period. In the stent patent group, all patients were relived from their symptoms without significant laterality of their circumference of both thighs, which was defined as "Relief of the symptom". In the stent occluded group, one patient obtained the "Relief of the symptom", however the other 
Table 4 Early complication and follow-up

\begin{tabular}{|c|c|c|c|c|c|}
\hline Patient No. & Early rethrombosis & Secondary procedure & Follow-up (months) & Stent patency & Outcome \\
\hline 1 & 5 days & Not performed & 12 & - & No change \\
\hline 2 & - & - & 13 & + & Relief \\
\hline 3 & - & - & 42 & + & Relief \\
\hline 4 & - & - & 1 & + & $\begin{array}{c}\text { Relief/Died } \\
\text { (Malignant disease) }\end{array}$ \\
\hline 5 & 7 days & Rethrombectomy & 15 & - & Relief \\
\hline 6 & - & _- & 30 & + & Relief \\
\hline 7 & - & - & 9 & + & Relief \\
\hline 8 & - & - & 9 & + & Relief \\
\hline
\end{tabular}

patient who had only once operation, was not recognized with significant relief of the symptoms, which was defined as 'No change of the symptom'.

\section{Discussion}

It is important that early thrombectomy for DVT can prevent long-term venous damage and PTS. ${ }^{10)}$ Therefore, invasive re-canalisation techniques have regained popularity and are considered to be a valid treatment option for iliofemoral DVT. ${ }^{10,11)}$ In the field of surgical thrombectomy, refinement and improvement of the operative technique as well as the combination with intra-operative angioplasty and stenting of the pelvic veins have made it possible to treat extensive DVTs involving the iliac veins from a small groin incision while still achieving complete re-canalisation of the venous system. ${ }^{3)}$ The results are good with mortality rates of $0 \%$ to $1 \%$, low morbidity rates, even if the rate of pulmonary embolism is $<1 \%,{ }^{9}$ and good long-term results for patency and valvular competence. ${ }^{3)}$ Recently, CDT has been used for the treatment of DVT; however, CDT results in a mean infusion time of 53 hours and major bleeding complications of $11 \%$ have been described. ${ }^{7)}$ CDT plus mechanical thrombectomy has mean infusion times of 23 hours and major bleeding complications of $14 \% .^{12)}$ In our series, surgical thrombectomy with isolated intra-operative thrombolysis had no major bleeding complications; therefore, surgical thrombectomy could be associated with a low rate of peri- and post-procedural risk for major bleeding complication compared to the alternative "less invasive" CDT approaches. ${ }^{9)}$

One of the main concerns with surgical thrombectomy was the risk of early rethrombosis. Even though one-year primary patency rate of $79 \%$ could only be achieved if no residual stenosis was persistent, residual stenosis of $1 \%-50 \%$ had a one-year patency rate of only $58 \%$ and residual stenosis of more than $50 \%$ had only $32 \% .{ }^{7)}$ MTS usually has stenotic lesion, therefore, we treat DVTs due to MTS with stents placement. The feasibility and success of procedure has been well documented. Nazarian, et al. demonstrated 4-year primary and secondary patency rates of $50 \%$ and $75 \%$ respectively in patients with iliac vein compression treated with thrombectomy, angioplasty, and stent placement. ${ }^{13)}$ AbuRahma, et al. demonstrated 1-year primary patency rates of $83 \%$ after combined thrombectomy, angioplasty, and stenting. ${ }^{14)}$ The use of thrombolysis and thrombectomy combined with iliac vein stenting has also been shown to improve patient outcomes and aid in the prevention of the sequlae of PTS. Patel, et al. employed Wallstent ${ }^{\mathrm{TM}}$ in 10 patients with MTS and reported complete resolution of all symptoms in all patients. They further reported that follow-up ultrasound revealed no evidence of valve insufficiency in the femoral or popliteal veins post-stenting. ${ }^{15)}$ As in our series, re-thrombosis only occurred within 1 week after thrombectomy. This was mainly due to missed residual stenosis or in some cases with thrombectomy on diseased veins after previous femoral DVT. These patients were not the best candidates for this technique. Furthermore, the postoperative anticoagulant therapy might be important for the stent patency. In our series, all patients who had iliac vein stenting were discharged on warfarin for 6 months. Most authors recommended less than 6 months anticoagulant therapy with warfarin after the stent placement. ${ }^{9,16,17)}$ It was reported that the mid-term patency of iliac vein stent placement was considerably higher, compared with the other medium size veins, such as the subclavian vein. ${ }^{18)}$ Because the pelvic stents were relatively immobile positioned, compared to the freely mobile subclavian vein. Therefore, we performed the warfarization simultaneously with heparinization postoperatively, with a target international normalized ratio of 2.0 for 6 months. 


\section{Conclusion}

We found that surgical thrombectomy with simultaneous stenting shows good results with acute symptomatic iliofemoral venous thrombosis. Stenting provided sufficient venous outflow and considerably might improve results in patients with underlying stenotic lesions, such as MTS. When MTS is diagnosed, aggressive endovascular management should be pursued to prevent thrombus recurrence and symptoms of PTS.

\section{Disclosure Statement}

Igari and other co-authors have no conflict of interest.

\section{References}

1) Hölper P, Kotelis D, Attigah N, et al. Longterm results after surgical thrombectomy and simultaneous stenting for symptomatic iliofemoral venous thrombosis. Eur J Vasc Endovasc Surg 2010; 39: 349-55.

2) Murphy EH, Davis CM, Journeycake JM, et al. Symptomatic ileofemoral DVT after onset of oral contraceptive use in women with previously undiagnosed May-Thurner syndrome. J Vasc Surg 2009; 49: 697-703.

3) Hartung O, Benmiloud F, Barthelemy P, et al. Late results of surgical venous thrombectomy with iliocaval stenting. J Vasc Surg 2008; 47: 381-7.

4) Ziegler S, Schillinger M, Maca TH, et al. Post-thrombotic syndrome after primary event of deep venous thrombosis 10 to 20 years ago. Thromb Res 2001; 101: 23-33.

5) May R, Thurner J. The cause of the predominantly sinistral occurrence of thrombosis of the pelvic veins. Angiology 1957; 8: 419-27.

6) Sharifi M, Mehdipour M, Bay C, et al. Endovenous therapy for deep venous thrombosis: the TORPEDO trial. Catheter Cardiovasc Interv 2010; 76: 316-25.

7) Mewissen MW, Seabrook GR, Meissner MH, et al. Catheter-directed thrombolysis for lower extremity deep venous thrombosis: report of a national multicenter registry. Radiology 1999; 211: 39-49.

8) Ishihara K, Hiromatsu S, Shintani Y, et al. Clinical outcome of perioperative nonpermanent vena cava filter placement in patients with deep venous thrombosis or blood stasis of the vein. Surg Today 2009; 39: 764-9.

9) Lindow C, Mumme A, Asciutto G, et al. Long-term results after transfemoral venous thrombectomy for iliofemoral deep venous thrombosis. Eur J Vasc Endovasc Surg 2010; 40: 134-8.

10) Comerota AJ, Paolini D. Treatment of acute iliofemoral deep venous thrombosis: a strategy of thrombus removal. Eur J Vasc Endovasc Surg 2007; 33: 351-60; discussion 361-2.

11) Comerota AJ, Gravett MH. Iliofemoral venous thrombosis. J Vasc Surg 2007; 46: 1065-76.

12) Vedantham S, Vesely TM, Parti N, et al. Lower extremity venous thrombolysis with adjunctive mechanical thrombectomy. J Vasc Interv Radiol 2002; 13: $1001-8$.

13) Nazarian GK, Bjarnason H, Dietz CA, et al. Iliofemoral venous stenoses: effectiveness of treatment with metallic endovascular stents. Radiology 1996; 200: 193-9.

14) AbuRahma AF, Perkins SE, Wulu JT, et al. Iliofemoral deep vein thrombosis: conventional therapy versus lysis and percutaneous transluminal angioplasty and stenting. Ann Surg 2001; 233: 752-60.

15) Patel NH, Stookey KR, Ketcham DB, et al. Endovascular management of acute extensive iliofemoral deep venous thrombosis caused by May-Thurner syndrome. J Vasc Interv Radiol 2000; 11: 1297-302.

16) Kim JY, Choi D, Guk Ko Y, et al. Percutaneous treatment of deep vein thrombosis in May-Thurner syndrome. Cardiovasc Intervent Radiol 2006; 29: 571-5.

17) Hurst DR, Forauer AR, Bloom JR, et al. Diagnosis and endovascular treatment of iliocaval compression syndrome. J Vasc Surg 2001; 34: 106-13.

18) Lumsden AB, MacDonald MJ, Isiklar H, et al. Central venous stenosis in the hemodialysis patient: incidence and efficacy of endovascular treatment. Cardiovasc Surg 1997; 5: 504-9. 